\title{
On isomorphism classes and invariants of a subclass of low-dimensional complex filiform Leibniz algebras
}

\begin{abstract}
The article aims to study the classification problem of low-dimensional complex filiform Leibniz algebras. It is known that filiform Leibniz algebras come out from two sources. The first source is a naturally graded non-Lie filiform Leibniz algebra, and another one is a naturally graded filiform Lie algebra. In this article, we classify a subclass of the class of filiform Leibniz algebras appearing from the naturally graded non-Lie filiform Leibniz algebra. We give complete classification and isomorphism criteria in dimensions 5-7. The method of classification is purely algorithmic. The isomorphism criteria are given in terms of invariant functions.
\end{abstract}

Keyword: Filiform Leibniz algebra; Invariant function; Isomorphism 